Revista Eletrônica de Farmácia

Eletronic Journal of Pharmacy

ISSN 1808-0804

doi 10.5216/ref.v16i0.45007

Artigo Original

\title{
Conhecimento e utilização de anticoncepção de emergência por jovens no brasil: revisão integrativa da literatura
}

\section{Knowledge and use of emergency contraception by young pe- ople in brazil: integrative review of literature}

\section{Conocimiento y utilización de anticoncepción de emergencia por jóvenes en brasil: una revisión integrativa de la literatura}

\author{
PORTO, Marta Soares ${ }^{1}$, AREDA, Camila Alves ${ }^{1}$, MEINERS, Micheline Marie M A ${ }^{1}$, GALATO, Dayani ${ }^{1}$, SILVA, Emília \\ Vitória da ${ }^{1 *}$ \\ ${ }^{1}$ Faculdade de Ceilândia, Universidade de Brasília - FCE/UnB \\ *emiliavitoria67@gmail.com
}

Resumo. Introdução: A pílula de anticoncepção de emergência (PAE) é um método seguro e eficaz de prevenção da gravidez não planejada e possui indicação após relação sexual desprotegida. Os jovens representam uma parcela importante entre usuários desse método contraceptivo. Investigar o nível de conhecimento, acesso e utilização da PAE é fator fundamental para nortear políticas públicas que favoreçam o bom uso desse medicamento. Objetivo: Realizar revisão integrativa da literatura de estudos descritivos transversais, com aplicação de questionário, que relatam a utilização e o conhecimento a respeito de PAE por jovens no Brasil. Método: Foram levantados artigos indexados nas bases de dados Lilacs, SciELO e Pubmed até o dia 30 de junho de 2015, por meio dos descritores "emergency contraception", "emergency contraception and Brazil" e "emergency contraceptive and Brazil". Resultados: Após exclusão dos artigos que não se enquadraram aos critérios estabelecidos, restaram nove estudos, dos quais foram extraídas informações relativas à publicação e seus principais resultados. A análise dos estudos mostra que $64 \%$ ou mais dos entrevistados tem conhecimento da PAE, adquiridos notadamente por meio de amigos. Entre 20 e 50\% dos entrevistados afirmaram já ter utilizado este tipo de anticoncepcional. Conclusão: Os dados reforçam a necessidade de nortear campanhas educativas dirigidas ao público jovem.

Palavras-chave: Anticoncepção pós-coito. Gravidez não planejada. Inquéritos epidemiológicos. Brasil.

\begin{abstract}
Introduction: Emergency contraception pill (EC) is a safe and effective method of preventing unplanned pregnancy and has indication after unprotected intercourse. Youngers represent an important portion of users of EC. Investigate the level of knowledge, access and use of EA is fundamental to guide public policies that favor the spread of this medicine. Objective: To conduct an integrative literature review of transversal descriptive study with a questionnaire, reporting the use and knowledge of EC in young people in Brazil. Method: The articles indexed on the databases Lilacs, SciELO and Pubmed until 30 June 2015 was raised, through the descriptors "emergency contraception", "emergency contraception and Brazil"and "emergency contraceptive and Brazil."Those who did not meet the inclusion criteria were excluded. Results: Nine studies were selected of which were extracted information about methodological aspects and its main results. Despite the heterogeneity of the studies reviewed here, the analysis shows that almost all of the respondents (at least 64\%) known about EC, acquired mainly through friends. 20 to $50 \%$ of respondents reported having used this contraceptive alternative. Conclusion: this data reinforces the idea of using the data summarized here to guide educational campaigns aimed at this audience.
\end{abstract}

Key-words: Contraception postcoital. Pregnancy unplanned. Health surveys. Brazil.

Resumen. Introducción: La píldora anticoncepcional de emergencia (PAE) es un método seguro y eficaz de prevención de embarazo no deseado que se usa después de la relación sexual no protegida. Los jóvenes representan parte importante de usuarios de este método anticonceptivo. Investigar el nivel de conocimiento, acceso y utilización de la PAE es fundamental para orientar políticas públicas que favorezcan el buen uso de este medicamento. Objetivo: Realizar una revisión integrativa de estudios descriptivos transversales, con aplicación de cuestionarios, que relaten la utilización y conocimiento sobre la PAE en jóvenes brasileños. Método: Se recopilaron artículos indexados en Lilacs, SciELO y Pubmed hasta el día 30 de junio del 2015, por medio de las palabras clave "emergency contraception", "emergency contraception and Brazil"y "emergency contraceptive and Brazil". Resultados: Después de excluir aquellos que no cumplieron con los criterios establecidos, quedaron nueve estudios, de los cuales se extrajeron informaciones relativas a la publicación y sus principales resultados. El análisis mostró que más del $64 \%$ de los entrevistados tiene conocimiento de la PAE adquirido por medio de amistades. Entre 20 y $50 \%$ de los entrevistados afirmaron que ya habían utilizado esta alternativa anticoncepcional. Conclusión: Esta información refuerza la idea de utilizar los datos aquí resumidos para guiar campañas educativas dirigidas a este público.

Palabras-clave: Anticoncepción poscoital. Embarazo no planeado. Encuestas epidemiológicas. Brasil. 


\section{Introdução}

A pílula de anticoncepção de emergência (PAE) é um importante método anticoncepcional indicado para prevenir gravidez após uma relação sexual desprotegida ou inadequadamente protegida.(1) Diferente de outros métodos contraceptivos mais comumente disponíveis, a PAE é utilizada após a relação sexual.(2,3) A mesma é conhecida popularmente como "pílula do dia seguinte" - nomenclatura equivocada, pois não é necessário esperar o "dia seguinte" para ser administrada, e pode ser utilizada até o quinto dia após a relação sexual.(4,5)

Existem três tipos de PAE, a pílula de levonorgestrel, o regime combinado de etinilestradiol e levonorgestrel (Yuzpe) e o que contém o acetato de ulipristal.(1) No Brasil, atualmente, apenas o primeiro tipo é comercializado e distribuído em unidades de atenção básica do Sistema Único de Saúde (SUS), nas formas de comprimidos de 0,75 mg e 1,5 mg.(2) Assim, para fins deste artigo, PAE estará se referindo somente a pílula contendo o levonorgestrel.

"Estudos mostram que o uso da PAE reduziu a probabilidade de gravidez após uma relação sexual 52 a 100\%". Contudo, essa efetividade diminui quanto maior o tempo transcorrido entre a relação sexual e seu uso. Mesmo sendo relativamente segura, a PAE pode provocar alteração do sangramento vaginal, náuseas e vômitos, dor de cabeça, dor abdominal, sensibilidade nas mamas, tontura e fadiga.(1)

A sexualidade na adolescência muitas vezes pode ser direcionada pelo processo de socialização e pelo acesso (ou falta dele) à informação, ao diálogo e aos cuidados que a sociedade oferece aos jovens.(6) Assim, pode-se considerar a falta de acesso à informação uma das principais causas de relações sexuais desprotegidas, contribuindo para a alta prevalência de gravidez na adolescência.(7) Dados oficiais mostram que 19,3\% das crianças nascidas vivas no Brasil, em 2010, foram de mães com idade inferior a 20 anos,(8) o que pode ser considerada uma taxa alta, tendo em vista o contexto socioeconômico brasileiro.

Visto que o acesso ao conhecimento sobre os métodos contraceptivos é um direito das mulheres, incluindo as adolescentes, ressalta-se a importância da orientação sobre a PAE dentro da proposta de educação sexual,(9) com vistas a promover o seu uso seguro e adequado e, assim, evitar sua utilização excessiva.

Dessa forma, a avaliação do grau de conhecimento e uso da PAE por jovens, em especial por indivíduos do sexo feminino, permite um melhor entendimento de sua utilização e fornece dados úteis sobre seu perfil farmacoepidemiológico. A partir dessas informações pode-se direcionar o desenvolvimento de medidas educativas para corrigir e melhorar o conhecimento a respeito do tema entre mulheres adolescentes e, consequentemente, promover um uso mais adequado desse medicamento.
A revisão integrativa é uma estratégia de pesquisa realizada por meio de busca e seleção de estudos experimentais e não-experimentais seguindo um método criterioso, quando se deseja obter um profundo entendimento de um determinado fenômeno com base em estudos anteriores.(10,11)

Neste sentido, os autores optaram por realizar uma revisão integrativa de estudos descritivos e observacionais que investigaram a utilização e o nível de conhecimento da anticoncepção de emergência por jovens no Brasil, com vistas a conhecer este cenário e nortear ações educativas envolvendo este público.

\section{Método}

Trata-se de uma revisão integrativa da literatura, realizada a partir de buscas nas bases de dados eletrônicas: Literatura Latino-Americana e do Caribe em Ciências da Saúde (Lilacs); Scientific Eletronic Library Online (SciELO) e Pubmed, desde o início da indexação até abril de 2015. Os descritores utilizados na busca foram os seguintes: na base Lilacs, "emergency contraception and Brazil", "emergency contraception" e "adolescente"; na base SciELO, "emergency contraception" sendo a pesquisa realizada para o contexto brasileiro; e, na base Pubmed, "emergency contraceptive and Brazil".

Dos artigos recuperados, foram excluídos aqueles que 1) não foram realizados no Brasil; 2) eram trabalhos teóricos ou de revisão; 3) não abordavam a temática da anticoncepção de emergência; 4) apresentavam outro desenho epidemiológico que não o transversal; 5) que incluíam exclusivamente outras populações que não jovens (no estudo considerou-se jovem o indivíduo com até 25 anos); e 6) não adotaram a aplicação de questionário.

Dos artigos selecionados, foram extraídas as informações: objetivos principais, tipo de estudo, público-alvo, tamanho da amostra, instrumento de coleta empregado, período de realização, ambiente de condução do estudo, localidade, ano de publicação e autores. Estas informações foram organizadas em tabelas e analisadas categoricamente, fundamentando-se nos resultados mais relevantes e frequentes a fim de melhor compreender o perfil de utilização e conhecimento sobre anticoncepção de emergência por jovens no Brasil.

Por se tratar de uma revisão da literatura e, portanto, não se caracterizar como um estudo envolvendo seres humanos, o projeto não foi submetido ao Comitê de Ética em Pesquisa (CEP).

\section{Resultados}

A Figura 1 apresenta o fluxograma seguido durante a revisão, por meio do qual se verifica que a busca nas bases de dados possibilitou a identificação inicial de 107 artigos, dos quais apenas nove não se enquadraram nos critérios de 


\begin{tabular}{|c|c|c|}
\hline \multicolumn{2}{|c|}{$\begin{array}{l}\text { Trabalhos localizados por } \\
\text { meio dos critérios definidos } \\
\text { para a busca } \\
(\mathrm{N}=107)\end{array}$} & \\
\hline | & $\begin{array}{l}\text { Excluídos por não } \\
\text { aos critérios de in }\end{array}$ & $\begin{array}{l}\text { enquadrarem } \\
\text { são }(\mathrm{N}=98)\end{array}$ \\
\hline \multicolumn{3}{|c|}{$\begin{array}{l}\text { Análise dos textos completos e } \\
\text { extração dos dados }(\mathrm{N}=9)\end{array}$} \\
\hline \multicolumn{3}{|c|}{$\begin{array}{l}\text { Figura 1. Fluxograma da inclusão e exclusão de artigos par } \\
\text { realização da revisão integrativa da literatura. }\end{array}$} \\
\hline $\begin{array}{r}\text { Objetivo princip } \\
\text { selecion }\end{array}$ & $\begin{array}{l}\text { al do estudo } \\
\text { lado }\end{array}$ & $\begin{array}{l}\text { Número de } \\
\text { artigos }\end{array}$ \\
\hline $\begin{array}{r}\text { Caracterizar o compor } \\
\text { contrace }\end{array}$ & $\begin{array}{l}\text { tamento sexual e } \\
\text { ptivo }\end{array}$ & 4 \\
\hline $\begin{array}{r}\text { Descrever a prevalên } \\
\text { perfil de utilização da } \\
\text { emergê }\end{array}$ & $\begin{array}{l}\text { cia de consumo e } \\
\text { anticoncepção de } \\
\text { ncia }\end{array}$ & 6 \\
\hline Conhecer os determi & nantes do uso da & 3 \\
\hline PAE & & \\
\hline $\begin{array}{r}\text { Identificar o nível de } \\
\text { PAE }\end{array}$ & conhecimento da & 7 \\
\hline
\end{tabular}

Tabela 1. Descrição e quantificação dos objetivos principais dos estudos analisados.

exclusão estabelecidos para a revisão.

Por meio do método utilizado para esta revisão, apesar de haver variabilidade entre os estudos transversais - por exemplo, quanto ao público-alvo, local do estudo e tipo de coleta de dados - realizou-se análise e comparação entre os artigos selecionados, levantando os objetivos principais, como pode ser observado na Tabela 1.

No Quadro 1 são sumarizadas as informações relevantes dos estudos selecionados, com destaque para o públicoalvo, faixa etária e instrumento utilizado.

Em relação ao público-alvo, todos eram estudantes, em sua maioria universitários (apenas três estudos foram realizados com estudantes colegiais); seis estudos trabalharam tanto com indivíduos do sexo masculino quanto do sexo feminino, enquanto três entrevistaram apenas pessoas do sexo feminino. A respeito do tamanho da amostra, a mesma foi

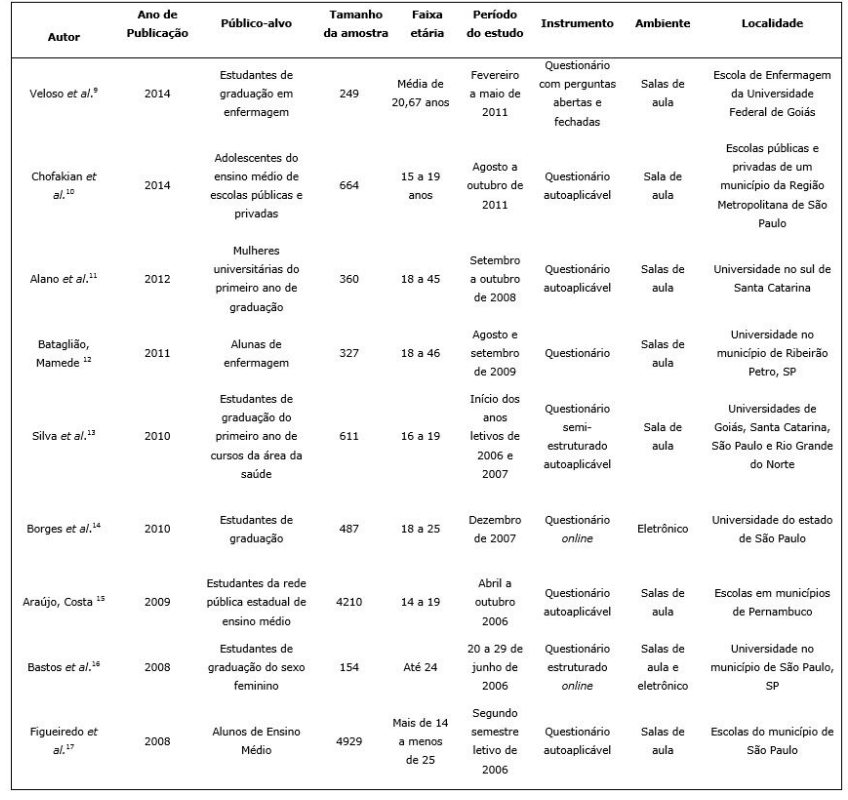

Quadro 1: Resumo das principais informações extraídas dos artigos.

diversificada, variando entre 154 e 4.929 entrevistados.

Todos os estudos investigaram a taxa de utilização da PAE entre os participantes e, de acordo com o que pode ser observado na Tabela 2, os valores de prevalência do uso variaram entre 21 e $50 \%$.

Apenas dois estudos retrataram a forma de uso da anticoncepção de emergência em relação ao tempo decorrido entre o ato sexual e a administração do medicamento. Na pesquisa de Alano et al. (2012)12, 97,1\% afirmaram ter feito uso do medicamento em até 72 horas após a relação sexual; dentre estes, a grande maioria $(87,1 \%)$ o fez em até 24 horas. Em contrapartida, os resultados de Araújo e Costa (2009)(13) foram apresentados de maneira diferente: utilização em até 72 horas foi de apenas $22,1 \%$, quando falta a menstruação $14,7 \%$, antes do ato sexual $46,9 \%$ e após as 72 horas $16,3 \%$.

Assim como acontece com os demais medicamentos, o uso adequado da PAE pressupõe que a usuária, efetiva ou em potencial, e seu parceiro, tenham um mínimo de informação sobre suas características e propriedades. Nos estudos analisados, esse nível de conhecimento prévio sobre a pílula esteve sempre acima de 60\% (Tabela 2). Entretanto, esse nível grau de conhecimento não é garantia de uso racional da pílula.

Em função da heterogeneidade dos questionários aplicados nos estudos envolvidos nesta revisão, nem todos avaliaram as fontes nas quais os estudantes obtiveram informação sobre a PAE. Somente quatro dos nove estudos 


\begin{tabular}{|c|c|c|}
\hline \multicolumn{3}{|c|}{ Conhecimento } \\
\hline Autores & prévio & Utilização PAE \\
\hline Veloso et al., 2014 & $96 \%$ & $29 \%$ \\
\hline $\begin{array}{c}\text { Chofakian et al., } \\
2014^{10}\end{array}$ & $100 \%$ & $26 \%$ \\
\hline Alano et al., $2012^{11}$ & $99 \%$ & $49 \%$ \\
\hline $\begin{array}{c}\text { Bataglião, Mamede, } \\
2011^{12}\end{array}$ & $100 \%$ & $21 \%$ \\
\hline Silva et al., $2010^{13}$ & $\begin{array}{c}H=97 \% / M=96 \% \\
(*)\end{array}$ & $42 \%$ \\
\hline Borges et al., $2010^{14}$ & - & $50 \%$ \\
\hline Araújo, Costa, $2009^{15}$ & $64 \%$ & $28 \%$ \\
\hline Bastos et al., $2008^{16}$ & - & $46 \%$ \\
\hline $\begin{array}{l}\text { Figueiredo et al., } \\
\qquad 2008^{17}\end{array}$ & $\begin{array}{c}M=92 \% / H=78 \% \\
(*)\end{array}$ & $37 \%$ \\
\hline
\end{tabular}

$\left.{ }^{*}\right)$ - Dados relativos a sexo masculino $(\mathrm{H})$ e sexo feminino (M).

Tabela 2. Taxa de conhecimento prévio sobre e utilização da pílula de anticoncepção de emergência por estudo.

\begin{tabular}{|c|c|c|c|c|}
\hline $\begin{array}{l}\text { Fonte de } \\
\text { informação }\end{array}$ & $\begin{array}{l}\text { Chofakian et } \\
\text { al. } 2014^{10} \text {; } \\
\left(^{*}\right)\left({ }^{* *}\right)\end{array}$ & $\begin{array}{l}\text { Bataglião, } \\
\text { Mamede, } \\
2011^{12 ;(* *)}\end{array}$ & $\begin{array}{l}\text { Araújo, } \\
\text { Costa, } \\
2009^{15}\end{array}$ & $\begin{array}{l}\text { Figueiredo } \\
\text { et al., } \\
2008^{17 ;(*)(* *)}\end{array}$ \\
\hline Escola/Faculdade & $37,8 \%$ & $65,1 \%$ & $11,8 \%$ & $48,7 \%$ \\
\hline Amigos & $48,3 \%$ & $61,2 \%$ & $15,6 \%$ & $65,2 \%$ \\
\hline Mídia & - & $63,6 \%$ & $6,0 \%$ & $47,4 \%$ \\
\hline Pais ou parentes & $25,9 \%$ & - & $15,7 \%$ & - \\
\hline $\begin{array}{l}\text { Profissionais de } \\
\text { saúde }\end{array}$ & $13,2 \%$ & - & $14,1 \%$ & $20,2 \%$ \\
\hline Farmácias & $6,1 \%$ & - & $3,3 \%$ & $16,1 \%$ \\
\hline
\end{tabular}

(*) Inclui respostas de indivíduos de ambos os sexos (masculino e feminino).

(**) Os entrevistados tiveram a liberdade de escolher mais de uma opção.

Tabela 3. Principais fontes de informação sobre anticoncepção de emergência por estudo.

fizeram essa avaliação, sendo as principais fontes de informação descritas na Tabela 3.

\section{Discussão}

Os resultados desta revisão demonstram que a PAE é uma estratégia bastante conhecida dos jovens avaliados e que entre um quinto e metade da população investigada nos estudos já utilizou esta alternativa contraceptiva. Contudo, os achados da presente pesquisa não podem ser extrapolados para outras populações, uma vez que todos os trabalhos foram desenvolvidos em ambientes educacionais (colégios ou universidades). Neste contexto, é possível supor que populações que não têm o mesmo acesso à educação podem apresentar conhecimento e atitudes referentes ao uso desta tecnologia de forma diferente daquela por ora apresentada. Além disso, o fato da maior parte dos sujeitos da pesquisa ser das regiões Sudeste e do Sul, também limita a extrapolação dos dados para outros jovens do Brasil.

Mesmo que existam limitações, pode-se constatar que os objetivos dos estudos aqui analisados apresentam similaridade, o que condiz com o propósito de associar seus resultados.

Observa-se que são estudos analisados foram publicados nos últimos nove anos e realizados entre 2006 e 2014, aplicando questionários em escolas e universidades de diferentes localidades brasileiras. O período em que os estudos foram realizados coincide com a época em que a PAE teve seu acesso ampliado no país, em especial no sistema público de saúde, por meio de medidas tomadas pelo Ministério da Saúde.(18)

Os questionários autoaplicáveis foram as ferramentas mais utilizadas nos estudos. Esse instrumento é conhecido por ser simples, de fácil aplicação e de baixo custo, (19) o que pode explicar o seu uso na totalidade dos estudos analisados. Ademais, por envolver assunto ligado à sexualidade, que ainda é tabu em nossa sociedade, esse tipo de instrumento de coleta de dados evita inibição dos respondentes ou até mesmo omissão de informações e mentiras.

Considerando a faixa etária estudada em cada estudo, houve algumas variações, pois enquanto grande parte das investigações utilizou faixa etária restrita de um público jovem (até 25 anos de idade), outros, apesar de ser minoria, incluíram adultos. De qualquer forma, esses últimos, por se enquadrarem nos critérios de estudo, foram incluídos na análise. E, ainda sobre esse aspecto, nota-se que a forma de apresentação dos valores não foi padronizada, pois, na tentativa de organizar os dados, dois estudos não definiram exatamente as idades mínimas e máximas dos entrevistados.

A anticoncepção de emergência foi utilizada pelas estudantes em proporções semelhantes nos estudos de Alano et al. (2012) (11), Silva et al. (2010)(13) e Bastos et al. (2008),(16) sendo a prevalência numericamente próxima a $45 \%$. Borges et al. (2010)(14) apresentaram o maior valor entre os estudos $(50 \%)$, mas é importante considerar que esse número resulta de entrevista feita com homens e mulheres, de modo que constatou-se não haver diferença estatisticamente significativa entre os $\operatorname{sexos}(p=0,963)$.

Já Bataglião e Mamede (2011)(12) apresentaram o menor índice de uso de PAE (20,8\%). Entretanto, enquanto os demais estudos usaram como denominador apenas aqueles estudantes sexualmente experientes, nesse trabalho não houve seleção quanto a este critério. Ademais, a pergunta limitava o período dos últimos doze meses que antecederam 
a data da entrevista para a resposta, como fizeram também Araújo e Costa (2009) (15), que também revelaram um baixo índice de uso de APE em comparação aos demais estudos $(27,5 \%)$.

Outro aspecto que pode ser considerado quanto à utilização de anticoncepção de emergência é a faixa etária do público entrevistado em cada estudo. Ao comparar-se os dois estudos que foram realizados com colegiais, Figueiredo et al. (2008)(17) e Araújo e Costa (2009)(15) com os demais, percebe-se que esses mostraram valores menores que aqueles apresentados pelo público universitário (com exceção de Bataglião e Mamede, 2011)(12). Figueiredo et al. (2008)(17) identificaram índice de 9,7\% e, ainda, observaram maior uso desse método conforme há aumento da faixa etária $(\mathrm{p}=0,0093)$.

No entanto, o mesmo não foi notado em todos os estudos, pois Alano et al. (2012)(11) constataram que quanto menor a idade, maior foi a prevalência de utilização do método. Tais controvérsias podem ser explicadas pelo apresentado na Pesquisa Nacional de Demografia e Saúde da Criança e da Mulher (PNDS 2006), que revela que maior índice de uso é observado nas idades entre 20 e 24 anos e menor entre os que se encontram na faixa etária compreendida entre 15 e 19 anos, (20) ou seja, há uma faixa etária que o uso é reduzido, o que pode ter influenciado na pesquisa de Figueiredo et al. (2008). (17) Ademais, os valores encontrados revelam taxa considerável de utilização da PAE, mas que apresentam variações conforme cada realidade.

Os resultados dos estudos analisados mostraram diferentes formas de utilização da pílula anticoncepcional de emergência, demonstrando o quão variado é o nível de conhecimento a respeito da PAE entre os estudantes. Dentre as consequências dessa variação pode estar uma gravidez indesejada devido ao uso inadequado do medicamento, uma vez que se sabe que a efetividade do fármaco depende do tempo transcorrido entre a relação sexual desprotegida e a administração do mesmo, sendo maior quanto mais precoce for o uso. Estudos estimaram que a efetividade do uso da pílula após 72 horas de uma relação sexual desprotegida pode ser menor que $15 \%$.(6)

Apesar de se poder considerar alto o grau de conhecimento sobre anticoncepção de emergência relatado nos estudos, destaca-se a elevada porcentagem $(36,1 \%)$ de mulheres que não conheciam a PAE no estudo de Araújo e Costa (2009).(15) Nos estudos que apresentaram o conhecimento de indivíduos de ambos os sexos, nota-se que enquanto em Silva et al. (2010)(13) essa diferença entre os sexos foi mínima (1,1\%), em Figueiredo et al. (2008)(17) foi relevante $(13,9 \%)$. Entretanto, em ambos os estudos foi verificado maior nível de conhecimento a respeito da PAE pelo sexo feminino. O estudo de Figueiredo et al. (2008)(17) revelou, ainda, menor conhecimento a respeito do fármacos em pessoas com até 15 anos de idade, independente do sexo.

Alguns fatores predizem um maior conhecimento sobre a PAE como: ser do sexo feminino, maior tempo de escolaridade e coabitação com os pais.(21) Portanto, estes resultados, em que as mulheres apresentavam maior escore de conhecimento, quando comparado aos homens, vão ao encontro do que dizem Chofakian et al. (2014).(10)

Observa-se que, com exceção dos índices de Araújo e Costa (2009), (15) os valores revelados são elevados, principalmente quando comparados com os apresentados pela Pesquisa Nacional de Demografia e Saúde da Criança e da Mulher (PNDS 2006),(20) que quantifica em pouco mais de $70 \%$ o conhecimento da PAE pela juventude feminina brasileira. Considerando-se tais valores é válido reforçar, como afirmado por Lefevre e Lefevre (2010),(22) que conhecer o método não significa saber como adquiri-lo e utilizá-lo e muito menos usar de forma adequada.

Algumas informações são fundamentais para o uso racional da PAE. Por exemplo, é importante saber que a mesma é indicada para uso após relação desprotegida, de preferência dentro do período de 72 horas depois do coito. Além disso, o método não deve ser utilizado por mulheres com porfiria aguda, doença arterial grave ou distúrbios tromboembolíticos ou alérgicas aos fármacos de sua composição. A PAE também é contraindicada durante a gravidez (categoria X).(23) No questionário aplicado por Silva et al. (2010), contudo, 40,7\% dos respondentes sabiam que o método pode ser utilizado até 72 horas após a relação sexual e $48 \%$ respondeu deveriam utilizar o fármaco de 24 a 48 horas após a relação sexual. Apenas $19 \%$ conheciam todas as indicações.(17)

Outro aspecto importante a ser considerado é o risco associado à utilização da PAE, principalmente aos relacionados ao uso repetitivo e às reações adversas. De acordo com o estudo de Figueiredo et al. (2008), 26,9\% dos entrevistados consideram o uso da PAE prejudicial à saúde, mas $16,9 \%$ acreditam que este método pode ser usado frequentemente.(20) Em outro estudo, 10\% das entrevistadas acreditavam que o método poderia ser usado todos os dias.(24)

Esses resultados mostram a importância da educação em saúde de jovens em idade reprodutiva quanto aos métodos contraceptivos. A PAE não deve substituir a pílula anticoncepcional convencional, uma vez que a primeira pode induzir alterações fisiológicas importantes e tem efetividade reduzida (52-94\%) quando comparada ao método convencional.(25)

Considerando a educação em saúde uma das atribuições clínicas do farmacêutico,(26) esse profissional pode auxiliar no desenvolvimento da autonomia e da responsabilidade das mulheres, e também de seus parceiros, em relação aos contraceptivos de emergência em ações diárias que envolvam o cuidado em saúde(27) fortalecendo a decisão de usar, ou não, a PAE. Dessa forma, neste contexto, pode ser proposta a implantação de um serviço clínico farmacêutico que englobe a educação em saúde para mulheres jovens estudantes do ensino médio ou universitárias.

As diferentes fontes de informação sobre a PAE foram relatadas em três estudos. As mesmas não foram muito homogêneas, pois de acordo com Araújo e Costa (2009)(15) 
os três principais meios de obtenção de informação foram pais ou parentes, seguido de amigos e profissionais de saúde; para Bataglião e Mamede (2011)(12) e Figueiredo et al. (2008)(17) os três meios mais frequentes foram escola/faculdade, mídia e amigos (Tabela 3). Em Araújo e Costa (2009)(15) e Bataglião e Mamede (2011), (12) os dados foram exclusivamente de entrevistadas do sexo feminino.

A mídia foi uma das fontes de informação mais relatadas, exceto em Araújo e Costa (2009),(15) já a escola/faculdade apresentou resultados bem expressivos em todos os estudos. Contudo, a fonte de informação mais prevalente foi aquela relacionada aos amigos, a única presente entre as três principais de cada estudo. Ainda considerando esse parâmetro, nota-se que dentre esses três estudos, o meio de obtenção de informação menos frequente foi a farmácia. Os serviços e profissionais de saúde também foram pouco citados, exceto em Araújo e Costa (2009),(15) no qual este meio de obtenção de informação esteve entre os três principais. A análise desses dados permite inferir que é importante se ater ao tipo de informação que circula sobre a PAE, visto que dentre as fontes citadas nos estudos a principal delas é a menos confiável.

O fato de a mais frequente fonte de informação ser os amigos reflete a prática de compartilhamento de experiência entre os jovens, o que contribui para o papel primordial que os pares têm nas questões que cercam a contracepção de emergência. (9)

É importante ressaltar que, nos estudos analisados, utilizou-se de informações retrospectivas, de modo que estas podem estar sujeitas ao viés de memória. Além disso, o fato dos estudos terem sido realizados com escolares e universitários e apenas em alguns locais faz com que a extrapolação dos dados não possa ser realizada a outras populações e nem a outras localidades.

Cabe aqui destacar que, para Galato e Corrêa (2011), o uso da PAE é considerado um aspecto de vulnerabilidade, pois de uma maneira geral o uso deste método anticoncepcional é realizado para prevenir a gravidez não planejada em situações em que há o coito não protegido. Neste caso, deixando o jovem vulnerável às doenças sexualmente transmissíveis. (28)

Esta observação demonstra e reforça a necessidade de elaboração de protocolos de pesquisa padronizados para esse público-alvo e tema, como sugerido no Fórum 2005: Adolescência e Contracepção de Emergência.(8) Isso facilitaria o reconhecimento do comportamento dos jovens frente à PAE por meio de avaliação de uso e dos seus desdobramentos. Estudos descritivos transversais, com aplicação de questionário, se mostram válidos e viáveis para retratar a situação do uso da anticoncepção de emergência entre a população jovem brasileira e, por isso, é importante que esses estudos continuem sendo realizados.

Dentre outras limitações desse trabalho podemos destacar a heterogeneidade dos estudos, o que dificultou uma consolidação dos dados; o caráter retrospectivo dos questionários, sempre sujeito a viés de memória; e a revisão ser do tipo integrativa e não sistemática, uma modalidade que poderia dar mais força as discussões. Além disso, dos estudos, apenas Chofakian et al. (2014)(10) avaliaram o aspecto religioso entre os entrevistados. O sentido e significado da sexualidade, e consequentemente o comportamento sexual, apresenta uma flexibilidade conforme a religião seguida pelo jovem.(29) Portanto, uma vez não considerado esse fator, pode haver viés de aferição e análise, uma vez que $\mathrm{o}$ aspecto da religiosidade pode afetar o comportamento sexual das pessoas.

\section{Conclusão}

A presente revisão integrativa traça o perfil da utilização e o nível de conhecimento acerca da PAE por usuárias e seus parceiros, apontando para seu uso frequente por jovens e, como as principais fontes de informação a seu respeito, os amigos. Os dados aqui consolidados podem servir de referência para planejamento de políticas públicas e ações educativas, estas últimas perfeitamente aplicáveis no cuidado farmacêutico. As políticas públicas poderiam ser voltadas a melhorar o acesso racional à PAE por meio da implementação de um serviço de acompanhamento das pacientes para evitar o uso repetitivo e incentivar a escolha de outro método de contracepção de uso contínuo, mais seguro e eficaz. Quanto às médicas educativas, estas podem ser planejadas e executadas focando, principalmente, nos efeitos adversos e nos riscos do uso frequente, de modo a promover o uso correto da pílula de anticoncepção de emergência.

\section{Referências}

1. Consorcio Latinoamericano de Anticoncepción de Emergencia (CLAE). Pílulas anticoncepcionais de emergência: orientações médicas e de prestação de serviços. São Paulo; 2015. $20 \mathrm{p}$.

2. Brasil. Ministério da Saúde. Anticoncepção de emergência: perguntas e respostas para profissionais da saúde. 2. ed. Brasília: Ministério da Saúde; 2011. 45 p.

3. Cavalcante MS. Perfil de utilização de contraceptivo de emergência a partir de um serviço de atendimento farmacêutico de uma rede de farmácias comunitárias (dissertação). Fortaleza: Universidade Federal do Ceará; 2009

4. Faúndes A, Brache V, Alvarez F. Emergency contraception-clinical and ethical aspects. Int $\mathrm{J}$ Gynecol Obstet. 2003; 82: 297-305.

5. World Health Organization. Emergency contraception. WHO Media Centre; July 2012. [citado em Ago 2015]. Disponível em http://www.who.int/mediacentre/ factsheets/fs244/en/. Acesso em: $11 \mathrm{dez} .2014$.

6. Heilborn, ML et al. Gravidez na adolescência e sexualidade: uma conversa franca comeducadores e educadoras. Centro Latino-Americano em Sexualidade e Direitos Humanos. Universidade do Estado do Rio de Janeiro. 2008. $48 \mathrm{p}$.

7. Figueiredo R, Bastos S, Telles JL. Perfil da distribuição da contracepção de emergência para adolescentes 
em municípios do estado de São Paulo. J Human Growth Develop. 2012; 22(1): 1-15.

8. Brasil. Ministério da Saúde. Saúde Brasil 2011: uma análise da situação de saúde e a vigilância da saúde da mulher. Brasília: Secretaria de Vigilância e m Saúde. Ministério da Saúde; 2012. 443 p.

9. 9. Saito MI, Leal MM. Adolescência e contracepção de emergência: Fórum 2005. Rev Paul Pediatria. 2007; 25(2): 180-6.

10. Biblioteca Prof. Paulo de Carvalho Mattos. Faculdade de Ciências Agronômicas. UNESP. Campus Botucatu. Tipos de revisão da literatura. Botucatu. 2015.

11. Souza MT, Silva MD, Carvalho R. Revisão integrativa: o que é e como fazer? Einstein 2010; 8(1 Pt 1): 102-6.

12. Alano GM, Costa LN, Miranda LR, Galato D. Conhecimento, consumo e acesso à contracepção de emergência entre mulheres universitárias no sul do Estado de Santa Catarina. Ciênc Saúde Col. 2012; 17(9): 2.397-404.

13. Araújo MSP, Costa LOBF. Comportamento sexual e contracepção de emergência entre adolescentes de escolas públicas de Pernambuco, Brasil. Cad Saúde Púb. 2009; 25(3): 551-62.

14. Veloso DLC, Peres VC, Lopes JSOC, Salge AKM, Guimarães JV. Anticoncepção de emergência: conhecimento e atitude de acadêmicos de enfermagem. Rev Gaúcha Enferm. 2014; 35(2): 33-9.

15. Chofakian CBN, Borges ALV, Fujimori E, Hoga LAK. Conhecimento sobre anticoncepção de emergência entre adolescentes do Ensino Médio de escolas públicas e privadas. Cad saude publica. 2014; 30(7): 1525-36.

16. Bataglião EML, Mamede FV. Conhecimento e utilização da contracepção de emergência por acadêmicos de enfermagem. Esc Anna Nery. 2011; 15(2): 284-90.

17. Silva FC, Vitalle MSS, Maranhão HS, Canuto MHA, Pires MMS, Fisberg M. Diferenças regionais de conhecimento, opinião e uso de contraceptivo de emergência entre universitários brasileiros de cursos da área de saúde. Cad Saúde Pública. 2010; 26(9):1821-1831.

18. Borges ALV, Fujimori E, Hoga LAK, Contin MV. Práticas contraceptivas entre jovens universitários: o uso da anticoncepção de emergência. Cad Saúde Pública. 2010; 26(4): 816-26.

19. Bastos MR, Borges ALV, Hoga LAK, Fernandes MP, Contin MV. Práticas contraceptivas entre jovens universitárias: o uso da anticoncepção de emergência. Texto Contexto Enferm. 2008; 17(3): 447-456.

20. Figueiredo R, Pupo LR, Segri NJ. Comportamento sexual e preventivo de adolescentes de São Paulo- um estudo com estudantes do Ensino Médio. BIS. 2008; 48: 31-33.

21. Souza RA, Brandão ER. Marcos normativos da anticoncepção de emergência e as dificuldades de sua institucionalização nos serviços públicos de saúde. Physis Rev Saude Col. 2009; 19(4): 1067-86.

22. Cosby PC. Métodos de pesquisa em ciências do comportamento. São Paulo: Editora Atlas; 2003.

23. Brasil. Ministério da Saúde. Formulário Terapêutico Nacional. Brasília, 2010. 1135 p.

24. Duarte CF, Holanda LB, Medeiros ML. Avaliação de conhecimento contraceptivo entre adolescentes grávidas em uma unidade básica de saúde do Distrito Federal. J Health Sci Inst. 2012; 30(2): 140-43.

25. WHO Media Centre Emergency contraception. Desenvolvido pela WHO Media Centre. Disponível em http://www.who.int/mediacentre/factsheets/fs244/en/. Acesso em: 11 out. 2018.

26. Brasil. Conselho Federal de Farmácia. Resolução $N^{\circ} 585 / 2013$. Regulamenta as atribuições clínicas do farmacêutico e dá outras providências. Brasília: CFF. 2013.

27. Brasil. Conselho Federal de Farmácia. Serviços farmacêuticos diretamente destinados ao paciente, à família e à comunidade. Brasília: CFF, 2016.

28. Galato D, Correia TS. Vunerabilidade das doenças sexualmente transmissíveis de pessoas vivendo em relacionamento estáveis em uma cidade do sul do Brasil. Arq Cat Medicina 2011; 40(2): 12-17.

29. Silva CG, Santos AO, Licciardi DC, Paiva V, Parker R. Religiosidade, juventude e sexualidade: entre a autonomia e a rigidez. Psicol Estud. 2008 ; 13(4): 683-92. 